\title{
MULTIPLIERS OF TRIGONOMETRIC SERIES AND POINTWISE CONVERGENCE $\left.{ }^{(}\right)$
}

\author{
BY \\ N. M. RIVIÈRE AND Y. SAGHER
}

Introduction. In a recent paper $\mathrm{M}$. Weiss and $\mathrm{A}$. Zygmund [7] have studied the pointwise convergence of a trigonometric series $\sum a_{n} e^{i n x}$ when the multipliers $\lambda_{n}=|n|^{i \gamma}(\gamma$ real $)$ are applied to it. The proof of their result makes use of Peano derivatives in $L^{p}$, which bear a close connection with the $t_{u}^{p}$ classes of A. P. Calderón and A. Zygmund [1]. In this paper we prove that conditions of Marcinkiewicz type for a multiplier are enough to preserve the $t_{u}^{p}$ classes (Theorems 1 and 2). As a consequence we obtain results on pointwise convergence for multipliers which satisfy a variational condition of Marcinkiewicz type (Theorem 3).

I. Notation. All functions to be considered in this paper are periodic with period $2 \pi$. We define

$$
\|f\|_{p}=\left(\int_{-\pi}^{\pi}|f(x)|^{p} d x\right)^{1 / p} \text { and } \mathscr{L}^{p}=\left\{f ;\|f\|_{p}<\infty\right\} .
$$

Definition 1. Let $u \geqq 0$, by $T_{u}^{p}\left(x_{0}\right), 1 \leqq p<\infty$; we denote the class of functions $f$, belonging to $\mathscr{L}^{p}$, and such that there exists a polynomial $P_{m}(x)$ of degree $m$, $m<u\left(P_{m}=0\right.$ if $\left.u=0\right)$, so that

$$
\left(\frac{1}{h} \int_{-h}^{h}\left|f\left(x-x_{0}\right)-P_{m}(x)\right|^{p} d x\right)^{1 / p} \leqq A h^{u},
$$

for $0<h \leqq \pi$, with $A$ independent of $h$. If $P_{m}(x)=\sum a_{n} x^{n}$, we write $T_{u}^{p}\left(x_{0}, f\right)=\|f\|_{p}$ $+\sum\left|a_{n}\right|+\inf \{A\}$.

Definition 2. Let $f \in T_{u}^{p}\left(x_{0}\right)$ we shall say that $f \in t_{u}^{p}\left(x_{0}\right)$ if and only if there exists a polynomial $P_{m}(x)$ of degree $m, m \leqq u$, such that

$$
\left(\frac{1}{h} \int_{-h}^{h}\left|f\left(x-x_{0}\right)-P_{m}(x)\right|^{p} d x\right)^{1 / p}=o\left(h^{u}\right) .
$$

$C^{\infty}$ will denote the class of infinitely differentiable functions.

II. Multipliers preserving $T_{u}^{p}\left(x_{0}\right)$ and $t_{u}^{p}\left(x_{0}\right)$. We start by stating some properties of the spaces $T_{u}^{p}\left(x_{0}\right)$ and $t_{u}^{p}\left(x_{0}\right)$ (see [1]):

(1) $T_{u}^{p}\left(x_{0}\right)$ is a Banach space with the norm $T_{u}^{p}\left(x_{0}, \cdot\right)$.

(2) $t_{u}^{p}\left(x_{0}\right)$ is a closed subspace of $T_{u}^{p}\left(x_{0}\right) ; C^{\infty}$ is dense in $t_{u}^{p}\left(x_{0}\right)$.

Received by the editors May 20, 1968.

(1) Research sponsored in part by the Office of Naval Research (NONR 3776(00)), and by NSF grant GP 8289. 
THEOREM 1. Let $k(x) \in \mathscr{L}^{1}$, such that:

(1) $\left|(d / d x)^{j} k(x)\right| \leqq C /|x|^{j+1}$ for $0 \leqq j \leqq r$.

(2) If $K(f)=(1 / \pi) \int_{-\pi}^{\pi} k(x-y) f(y) d y$, then $\|K f\|_{p} \leqq C\|f\|_{p}$.

Then $K$ is a continuous operator from $T_{u}^{p}\left(x_{0}\right)$ to $T_{u}^{p}\left(x_{0}\right)$, and from $t_{u}^{p}\left(x_{0}\right)$ to $t_{u}^{p}\left(x_{0}\right)$, for $u \leqq r$. Moreover $T_{u}^{p}\left(x_{0} ; K f\right) \leqq B_{u} C T_{u}^{p}\left(x_{0}, f\right)$, where $B_{u}$ is a constant depending on $u$ only.

Proof. The proof is similar to that of Lemma 5.1 in [1].

Without loss of generality we may assume that $x_{0}=0$. We will show first the preservation of $T_{u}^{p}\left(x_{0}\right)$.

Let $P_{m}(x)$ be the polynomial of (1.1) for $f(x)$.

Take $\phi(x) \in C^{\infty}$ such that: $\phi(x)=1$ for $|x|<\pi / 4$ and $\phi(x)=0$ for $|x|>\pi / 2$. Set $f(x)=f_{1}(x)+f_{2}(x)$ where $f_{2}(x)=P_{m}(x) \phi(x)$. Since for $h \leqq \pi / 4$,

$$
\int_{-h}^{h}\left|f_{2}(x)-P_{m}(x)\right|^{p} d x=0
$$

then it is clear that $T_{u}^{p}\left(0, f_{2}\right) \leqq B_{u} T_{u}^{p}(0, f)$.

On the other hand, if $\psi(x) \in C^{\infty}$,

$$
K(\psi)(x)=\frac{1}{\pi} \int_{-\pi}^{\pi} k(y)[\psi(x-y)-\psi(x)] d y+\frac{\psi(x)}{\pi} \int_{-\pi}^{\pi} k(y) d y
$$

Using then the fact that $|k(y)| \leqq C /|y|$ and that $\left|\int_{-\pi}^{\pi} k(y) d y\right| \leqq C$ (which follows from condition (2)); it follows that $|K(\psi)(x)| \leqq B_{\psi} C$. Similarly $\left|(d / d x)^{j} K(\psi)(x)\right|$ $\leqq B_{\psi} C$. Hence $T_{u}^{p}(0, K(\psi)) \leqq B_{\psi} C$. Applying this observation to $x^{n} \phi(x)$ :

$$
T_{u}^{p}\left(0, K\left(f_{2}\right)\right) \leqq \sum_{n<u}\left|a_{n}\right| T_{u}^{p}\left(0, K\left(x^{n} \phi\right)\right) \leqq B_{u} C T_{u}^{p}(0, f) .
$$

We pass now to consider $f_{1}(x)$. Clearly

$$
\left(\frac{1}{h} \int_{-h}^{h}\left|f_{1}(x)\right|^{p} d x\right)^{1 / p} \leqq T_{n}^{p}(0, f) h^{u}, \text { for } h \leqq \frac{\pi}{4}
$$

Claims:

$$
\begin{gathered}
\int_{-h}^{h}\left|f_{1}(x)\right||x|^{-j} d x \leqq B_{u} T_{u}^{p}(0, f) h^{u+1-j}, \text { for } 1 \leqq j<u+1 . \\
\int_{\pi \geqq|x| \geqq n}\left|f_{1}(x)\right||x|^{-j} d x \leqq B_{u} T_{u}^{p}(0, f) h^{u+i-j}, \text { for } u+1 \leqq j .
\end{gathered}
$$

We postpone the proof of (1.4) and (1.5) and proceed to show that $T_{u}^{p}\left(0, K\left(f_{1}\right)\right)$ $\leqq B_{u} C T_{u}^{p}(0, f)$. Expanding $k(x)$ by Taylor's formula, we have 


$$
\begin{aligned}
K\left(f_{1}\right)= & \frac{1}{\pi} \int_{-h}^{h} k(x-y) f_{1}(y) d y+\sum_{n<u} \frac{x^{n}}{n !} \frac{1}{\pi} \int_{-\pi}^{\pi}\left(\frac{d}{d y}\right)^{n} k(-y) f_{1}(y) d y \\
& +\frac{x^{l}}{l !} \frac{1}{\pi} \int_{h \leqq|x| \leqq \pi}\left(\frac{d}{d y}\right)^{l} k(\theta x-y) f_{1}(y) d y \\
& -\sum_{n<u} \frac{x^{n}}{n !} \frac{1}{\pi} \int_{-h}^{n}\left(\frac{d}{d y}\right)^{n} k(-y) f_{1}(y) d y
\end{aligned}
$$

where $u \leqq l<u+1$. Set $b_{n}=(1 / n ! \pi) \int_{-\pi}^{\pi}(d / d y)^{n} k(-y) f_{1}(y) d y$; then using condition (1) and (1.4):

$$
\left|b_{n}\right| \leqq B_{u} \int_{-\pi}^{\pi}\left|f_{1}(y)\right||y|^{-n-1} d y \leqq B_{u} C T_{u}^{p}(0, f) \quad(0 \leqq n<u) .
$$

Moreover

$$
\int_{-h / 2}^{h / 2}\left|\int_{-h}^{h} k(x-y) f_{1}(y) d y\right|^{p} d x \leqq C^{p} \int_{-h}^{h}\left|f_{1}(y)\right|^{p} d y \leqq C^{p} T_{n}^{p}(0, f) h^{p u+1}
$$

and for $n<u$

$$
\begin{array}{rl}
\int_{-h / 2}^{h / 2} \mid \frac{x^{n}}{n ! \pi} \int_{-h}^{h}\left(\frac{d}{d y}\right)^{n} & \left.k(-y) f_{1}(y) d y\right|^{p} d x \\
& \leqq B_{u} C^{p} \int_{-h / 2}^{h / 2}|x|^{n p}\left(\int_{-h}^{h}\left|f_{1}(y)\right||y|^{-n-1} d y\right)^{p} d x \\
& \leqq B_{u} C^{p} T_{u}^{p}(0, f) h^{u p+1} .
\end{array}
$$

Finally using (1.5)

$$
\begin{aligned}
\int_{-h / 2}^{h / 2}\left|\frac{x^{l}}{l !} \int_{\pi \geqq|x|>h}\left(\frac{d}{d y}\right)^{l} K(\theta x-y) f_{1}(y) d y\right|^{p} d x \\
\quad \leqq B_{u} C^{p} \int_{-h / 2}^{h / 2}|x|^{l p}\left(\int_{\pi \geqq|x|>h}\left|f_{1}(y)\right||y|^{-l-1} d y\right)^{p} d x \\
\leqq B_{u} C^{p} T_{u}^{p}(0, f) h^{u p+1} .
\end{aligned}
$$

From (1.6), (1.7), (1.8), (1.9) and (1.10) it follows that $T_{u}^{p}\left(0, K\left(f_{1}\right)\right) \leqq B_{u} C T_{u}^{p}(0, f)$, this inequality together with (1.3) proves the first part of the theorem.

For the second part it is enough to observe that if $f \in C^{\infty}$, then $K(f) \in C^{\infty}$, and $C^{\infty}$ is dense in $t_{u}^{p}\left(x_{0}\right)$.

Proof of Claims (1.4), (1.5). Assume that $\left(\int_{-h}^{h}|f(x)|^{p} d x\right)^{1 / p} \leqq A h^{u+1 / p}$; set $g(t)$ $=\int_{-t}^{t}|f(x)| d x \leqq A t^{u+1}$. Then for $0 \leqq j<r-1$ :

$$
\begin{aligned}
\int_{-h}^{h}|f(x)||x|^{-j} d x & =\lim _{\varepsilon \rightarrow 0} \int_{\varepsilon}^{h} t^{-j} d(g(t))=\lim _{\varepsilon \rightarrow 0}\left(\left.t^{-j} g(t)\right|_{\varepsilon} ^{h}+j \int_{\varepsilon}^{h} g(t) t^{-j-1} d t\right) \\
& \leqq A h^{u+1-j}+A \int_{0}^{h} t^{u-j} d t \leqq B_{u} A h^{u+1-j},
\end{aligned}
$$

and (1.4) follows. (1.5) can be proved using a similar argument. 
We shall discuss next what conditions on the Fourier coefficients of $k(x)$ guarantee properties (1) and (2).

LEMMA. Let $\left\{\lambda_{n}\right\}_{n=-\infty}^{+\infty}$, such that

(a) $\lambda_{n}=0$ for $|n|>N$,

(b) $\left|\lambda_{n}\right|<C$ and $\sum_{ \pm 2^{k}}^{ \pm 2^{k+1}}|n|^{r}\left|\Delta^{r+1}\left(\lambda_{n}\right)\right| \leqq C, r \geqq 1 \quad(k=0,1,2, \ldots)$

$$
\Delta \lambda_{n}=\lambda_{n}-\lambda_{n+1} ; \quad \Delta^{r} \lambda_{n}=\Delta\left(\Delta^{r-1} \lambda_{n}\right) .
$$

Then $k(x)=\sum_{-\infty}^{\infty} \lambda_{n} e^{i n x}$ satisfies property (1) for $j \leqq r-1$, and property (2).

Proof. Condition (b) implies

$$
\left|\Delta^{k} \lambda_{n}\right| \leqq B C|n|^{-k} \text { when } k \leqq r .
$$

Property (2) is now a consequence of the Marcinkiewicz multiplier theorem (see [3], [9, II, p. 232]) since

$$
\sum_{ \pm 2^{k}}^{ \pm 2^{k+1}}\left|\Delta \lambda_{n}\right| \leqq B C \sum_{ \pm 2^{k}}^{ \pm 2^{k+1}} \frac{1}{|n|} \leqq B C
$$

and therefore $\|K(f)\|_{p} \leqq B_{p} C\|f\|_{p}$ where $B_{p}$ depends on $p$ only. To prove property (b), set

$$
Z_{n}^{[k 1}(x)=\frac{e^{i(n+k) x}}{\left(e^{i x}-1\right)^{k}}
$$

observe that

$$
Z_{n}^{[k]}(x)-Z_{n+1}^{[k]}(x)=Z_{n+1}^{[k-1]}(x)
$$

and

$$
\left|Z_{n}^{[k]}(x)\right| \leqq 1 /|x|^{k}
$$

Set, for $x \neq 0, m=[1 /|x|]$ the integer part of $1 /|x|$, then, using (1.12) and summation by parts,

$$
\begin{aligned}
\left(\frac{d}{d x}\right)^{j} k(x) & =\sum_{n=-N}^{\infty}(i n)^{j} \lambda_{n} e^{i n x}=\sum_{n=-N}^{\infty} \Delta^{j+2}\left(\lambda_{n}(i n)^{j}\right) Z_{n}^{[j+2]}(x) \\
& =\sum_{|n| \leqq M} \Delta^{j+2}\left(\lambda_{n}(i n)^{j}\right) Z^{[j+2]}(x)+\sum_{|n|>M} \Delta^{j+2}\left(\lambda_{n}(i n)^{j}\right) Z_{n}^{[j+2]}(x) \\
& =P+Q .
\end{aligned}
$$

To estimate $Q$, let $2^{s} \leqq M \leqq 2^{s+1}$. Using the estimates (1.11), (1.13) and condition (b):

$$
\begin{aligned}
|Q| & \leqq \frac{B}{|x|^{j+2}} \sum_{k=s}^{\infty}\left\{\sum_{n= \pm 2^{k}}^{ \pm 2^{k+1}}\left(\sum_{l=0}^{j+2}\left|\Delta^{j+2-l}\left(\lambda_{n}\right)\right|\left(|n|^{j-l}\right)\right)\right\} \\
& \leqq \frac{B C}{|x|^{j+2}} \sum_{k=s}^{\infty} 2^{-k} \leqq \frac{B C}{|x|^{j+1}} .
\end{aligned}
$$


To estimate $P$, use a summation by parts argument and (1.12):

$$
\begin{aligned}
P=\sum_{n=-M}^{M+2} \Delta^{j}\left(\lambda_{n}(i n)^{j}\right) Z_{n}^{[j]}(x)- & \left\{\left[Z_{M+2}^{[j+1]}(x)-Z_{-M}^{[j+1]}(x)\right] \Delta^{j+1}\left(\lambda_{M+2}(i(M+2))^{j}\right)\right. \\
+ & \left.Z_{-M}^{[j+1]}(x)\left[\Delta^{j}\left(\lambda_{-M}(i M)^{j}\right)-\Delta^{j}\left(\lambda_{M+1}(i(M+1))^{j}\right)\right]\right\} \\
+ & \left\{\left[Z_{M+1}^{[j+2]}(x)-Z_{-M}^{[j+2]}(x)\right] \Delta^{j+1}\left(\lambda_{M+1}(i(M+1))^{j}\right)\right. \\
& \left.+Z_{-M}^{[j+2]}(x)\left[\Delta^{j+2}\left(\lambda_{-M}(-M)^{j}\right)-\Delta^{j+2}\left(\lambda_{M}(i M)^{j}\right)\right]\right\} .
\end{aligned}
$$

Hence using (1.11) and (1.13)

$$
|P| \leqq B C \frac{M}{|x|^{j}}+\frac{B C}{|x|^{j+1}}+\frac{B C}{M|x|^{j+2}} \leqq \frac{B C}{|x|^{j+1}} .
$$

The lemma follows. As a consequence of the lemma we have

THEOREM 2. Let $\left\{\lambda_{n}\right\}_{n=-\infty}^{\infty}$ such that $\left|\lambda_{n}\right| \leqq C$ and

$$
\sum_{ \pm 2^{k}}^{ \pm 2^{k+1}}\left|\Delta^{r+1}\left(\lambda_{n}\right)\right||n|^{r} \leqq C, \quad r \geqq 1 \quad(k=0,1,2, \ldots) .
$$

Define for $f \in C^{\infty}, f(x)=\sum a_{n} e^{i n x}, \wedge(f)=\sum_{n=-\infty}^{\infty} \lambda_{n} a_{n} e^{i n x}$. Then, for $u \leqq r-1$ $T_{u}^{p}\left(x_{0}, \wedge f\right) \leqq B_{u, p} C T_{u}^{p}\left(x_{0}, f\right)$, and as a consequence $\wedge$ can be extended to be $a$ continuous mapping from $t_{u}^{p}\left(x_{0}\right)$ into $t_{u}^{p}\left(x_{0}\right)$. (Since $C^{\infty}$ is dense in $t_{u}^{p}\left(x_{0}\right)$.)

Proof. Let $\phi(t) \in C^{\infty}(-\infty, \infty)$, such that $\phi(t)=1$ for $|t| \leqq 1$ and $\phi(t)=0$ for $|t| \geqq 2$. Set $\mu_{n}=\lambda_{n} \phi(n / N)$ where $N$ is a positive integer. Then, for $f \in C_{\infty}$ as before,

$$
\bigwedge_{N}(f)=\sum \mu_{n} a_{n} e^{i n x}=\frac{1}{\pi} \int_{-\pi}^{\pi} k_{N}(x-y) f(y) d y,
$$

where $k_{N}(x)=\sum_{n=-2 N}^{2 N} \mu_{n} e^{i n x}$. The theorem becomes an immediate consequence of Theorem 1 and the lemma once we observe that:

(i) Since $\left|(d / d x)^{i} \phi(x / N)\right| \leqq B / N$ for $|x| \leqq 2 N$ and it vanishes for $|x| \geqq 2 N$, then

$$
\left|\mu_{n}\right| \leqq B C \text { and } \sum_{ \pm 2^{k}}^{ \pm 2^{k+1}}\left|\Delta^{r+1}\left(\mu_{n}\right)\right||n|^{r} \leqq B C .
$$

(ii) For $f \in C^{\infty}$; $\bigwedge_{N}(f)$ converges uniformly to $\bigwedge(f)$ together with any finite number of derivatives; therefore $T_{u}^{p}\left(x_{0},\left(\bigwedge-\bigwedge_{N}\right)(f)\right) \rightarrow 0$ as $N \rightarrow \infty$.

III. Applications to pointwise convergence. Before we discuss the applications we shall introduce some of the notation to be used in this section.

Given a sequence $\left\{s_{n}\right\}_{n=0}^{\infty}$, define

$$
s_{n}^{(0)}=s_{n}, \quad s_{n}^{(j+1)}=\sum_{k=0}^{n} s_{k}^{(j)}
$$

and

$$
A_{n}^{(0)}=1, \quad A_{n}^{(j+1)}=\sum_{k=0}^{n} A_{k}^{(j)}
$$


$\sigma_{n}^{(k)}=s_{n}^{(k)} / A_{n}^{(k)}$ are the Cesàro means of $\left\{s_{n}\right\}$. If $\sigma_{n}^{(k)} \rightarrow s$ as $n \rightarrow \infty$ we shall say that $s_{n}$ is summable $(C, k)$. Finally we say that $s_{n}$ is summable Abel if

$$
\lim _{x \rightarrow 1^{-}} \sum_{n=0}^{\infty} s_{n} x^{n}
$$

exists.

As an application of our results of $\S \mathrm{I}$ we state

THEOREM 3. Let $\left\{\lambda_{n}\right\}_{n=-\infty}^{\infty}$ be a sequence satisfying $\left|\lambda_{n}\right| \leqq C$;

$$
\sum_{ \pm 2^{k}}^{ \pm 2^{k+1}}|n|^{r}\left|\Delta^{r+1}\left(\lambda_{n}\right)\right| \leqq C \quad(k=0,1,2, \ldots)
$$

and let $\sum_{n=-\infty}^{\infty} a_{n} e^{i n x}$ be a trigonometric series, summable $(C, k)$ on a measurable set $E$.

Then the series $\sum_{n=-\infty}^{\infty} \lambda_{n} a_{n} e^{i n x}$ is summable $(C, k)$ a.e. in $E$, provided $0 \leqq k \leqq r-2$.

The proof of the theorem will be divided into three lemmas.

LEMMA 1 (M. WeIss). If $\sum_{n=0}^{\infty} a_{n} e^{i n x}$ is summable $(C, k)$ on a measurable set $E$, then the $(k+1)$ th termwise integrated series

$$
f(x) \sim \sum_{0}^{\infty}(\text { in })^{-(k+1)} a_{n} e^{i n x} \in t_{k+1}^{p}(x)
$$

for almost every $x \in E(1 \leqq p<\infty)$.

For the proof of Lemma 1 we refer the reader to [6, Theorem $\left.C^{\prime}\right]$.

Lemma 2. If $g(x)=\sum_{n=-\infty}^{\infty} b_{n} e^{i n x} \in t_{k+1}^{p}(x)$ for $x \in E$, then the $(k+1)$ th termwise derivative of the series is

(i) summable $(C, k+1)$ a.e. in $E$,

(ii) summable $(C, k+3)$ everywhere in $E$.

Proof. According to the corollary of Theorem 9 in [1], for any closed set $F \subset E$, we may decompose $g=g_{1}+g_{2} ; g_{1}, g_{2} \in \mathscr{L}^{p}$; where

(a) $g_{1}$ has a classical $(k+1)$ th $\left(\mathscr{L}^{\infty}\right)$ Peano derivative at every point of $F$,

(b) $\left((1 / h) \int_{\left|x-x_{0}\right|<h}\left|g_{2}(x)\right|^{p} d x\right)^{1 / p}=O\left(h^{k+1}\right)$ and

$$
\int_{-\pi}^{\pi} \frac{\left|g_{2}(x)\right|}{\left|x-x_{0}\right|^{k+2}} d x<\infty
$$

(see [9, Theorem 10]).

If we now write the Fourier series expansion of $g_{1}$ and $g_{2}$ from property (a) it is known that the $(k+1)$ th termwise derivative of the Fourier series of $g_{1}$ is $(k+1)$ th Cesàro summable almost everywhere in $F$. (See [9, II, Theorem (5.4), p. 81].) 
From property (b) and the fact that if $K_{n}^{r}(t)$ is the $n$th Cesàro kernel of the $r$ means,

$$
\left|\left(\frac{d}{d t}\right)^{r} K_{n}^{r}(t)\right| \leqq \frac{C}{|t|^{r+1}} \quad(|t| \leqq \pi)
$$

(see [9, II, p. 60]). The result follows for $g_{2}$ and hence for $g$, proving (i). The statement (ii) is a consequence of the fact that the primitive of $g(x)$ has a $(k+2)$ th Peano derivative at every point of $E$ and hence the $(k+2)$ th termwise derivative of its Fourier series expansion is summable $(C, \alpha)$ for every $\alpha>k+2$. (See [9, II, Theorem (1.7), p. 60].)

The following lemma is due to A. Zygmund [8], [7].

LEMMA 3. Let $\left\{\lambda_{n}\right\}$ be a sequence satisfying $\left|\lambda_{n}\right| \leqq C ;\left|\Delta^{k+1}\left(\lambda_{n}\right)\right| \leqq C n^{-(k+1)}$. Set $s_{n}=\sum_{j=0}^{n} u_{j}$. If $s_{n}^{(k)}=o\left(n^{k}\right)$ then for $N=[1 /(1-x)]$,

$$
\sum_{n=0}^{\infty} \lambda_{n} u_{n} x^{n}-\sum_{n=0}^{N} s_{n}^{(k)} \Delta^{(k+1)}\left(\lambda_{n}\right) \rightarrow 0
$$

as $x \rightarrow 1^{-}$.

Proof. A summation by parts argument shows that

$$
\begin{aligned}
\sum_{n=0}^{\infty} \lambda_{n} u_{n} x^{n} & =\sum_{n=0}^{\infty} s_{n}^{(k)} \Delta^{(k+1)}\left(\lambda_{n} x^{n}\right) \\
& =\sum_{n=0}^{\infty} s_{n}^{(k)}\left\{\sum_{j=0}^{k+1}\left(\begin{array}{c}
k+1 \\
j
\end{array}\right) \Delta^{j}\left(x^{n}\right) \Delta^{k+1-j}\left(\lambda_{n+j}\right)\right\} \\
& =\sum_{n=0}^{\infty} s_{n}^{(k)} \Delta^{k+1}\left(\lambda_{n}\right) x^{n}+\sum_{j=1}^{k+1}\left(\begin{array}{c}
k+1 \\
j
\end{array}\right)(1-x)^{j}\left\{\sum_{n=0}^{\infty} s_{n}^{(k)} \Delta^{k+1-j}\left(\lambda_{n}\right) x^{n}\right\}
\end{aligned}
$$

Set $N=[1 /(1-x)]$, then

$$
\begin{aligned}
\sum_{n=0}^{\infty} \lambda_{n} u_{n} x^{n}- & \sum_{n=0}^{N} s_{n}^{(k)} \Delta^{k+1}\left(\lambda_{n}\right) \\
& =\sum_{n=0}^{N} s_{n}^{(k)}\left[\Delta^{k+1} \lambda_{n}\right]\left(x^{n}-1\right)+\sum_{n=N+1}^{\infty} s_{n}^{(k)} \Delta^{k+1}\left(\lambda_{n}\right) x^{n} \\
& +\sum_{j=1}^{k+1}\left(\begin{array}{c}
k+1 \\
j
\end{array}\right)(1-x)^{j}\left\{\sum_{n=0}^{\infty} s_{n}^{(k)} \Delta^{k+1-j}\left(\lambda_{n}\right) x^{n}\right\} \\
& =\sum_{n=1}^{N} o(1)(1-x)+o\left(\frac{1}{N}\right) \sum_{n=N}^{\infty} x^{n}+\sum_{j=1}^{k+1}(1-x)^{j} o\left((1-x)^{-j}\right)=o(1),
\end{aligned}
$$

since $\left|\Delta^{j}\left(\lambda_{n}\right)\right| \leqq C n^{-j}$ for $1 \leqq j \leqq k+1$.

REMARK. Since $\sum_{n=0}^{\infty} s_{n}^{(k)} \Delta^{k+1}\left(\lambda_{n}\right)$ and $\sum_{n=0}^{\infty} u_{n} \lambda_{n}$ are equisummable $(C, k)$, under the conditions of Lemma 3, we have that if the series $\sum \lambda_{n} u_{n}$ is summable Abel then it is also summable $(C, k)$. 
Proof of Theorem 3. We observe first (see [4] and [9, II, p. 216]) that if $\sum_{n=-\infty}^{\infty} a_{n} e^{i n x}$ is summable $(C, k)$ in $E$, then the conjugate series $\sum_{n=-\infty}^{\infty} \operatorname{sgn}(n) a_{n} e^{i n x}$ is summable $(C, k)$ a.e. in $E$. Hence without loss of generality we may assume that $a_{n}=0$ for $n<0$.

Set $f(x) \sim \sum_{n=0}^{\infty} a_{n}(i n)^{-(k+1)} e^{i n x}$, using Lemma $1, f(x) \in t_{k+1}^{p}(x)$ for almost every $x$ in $E(1 \leqq p<\infty)$. Applying Theorem $2, \bigwedge(f) \sim \sum_{n=0}^{\infty} \lambda_{n}(i n)^{-(k+1)} a_{n} e^{i n x}$ belongs to $t_{k+1}^{p}(x)$ a.e. in $E$.

From Lemma $2, \sum_{n=0}^{\infty} \lambda_{n} a_{n} e^{i n x}$ is summable $(C, k+1)$ a.e. in $E$ and therefore summable Abel a.e. in $E$.

The theorem follows by applying the remark to Lemma 3.

As a consequence of Theorem 3 we obtain a recent result of $M$. Weiss and A. Zygmund when $\lambda_{n}=|n|^{i \gamma}$ ( $\gamma$ real), see [7], also [5].

Another interesting application is

THEOREM 4. Let $F(t)$ be a bounded infinitely differentiable function on the real line and entire (real analytic) at infinity. Then $\lambda_{n}=F(n)$ is a multiplier sequence that preserves $(C, k)$ summability almost everywhere, for every $k \geqq 0$. This is so because $\left|(d / d t)^{n} F(t)\right| \leqq C_{n}|t|^{-n}$. In particular $F(t)$ being the bounded ratio of two polynomials will satisfy the conditions of Theorem 4.

\section{REFERENCES}

1. A. P. Calderón and A. Zygmund, Local properties of solutions of elliptic partial differential equations, Studia Math. 20 (1961), 171-225.

2. J. J. Marcinkiewicz, Sur les multiplicateurs des series de Fourier, Studia Math. 8 (1939), 78-91.

3. J. J. Marcinkiewicz and A. Zygmund, On the differentiability of functions and summability of trigonometric series, Fund. Math. 26 (1936), 1-43.

4. A. Plessner, On conjugate trigonometric series, Dokl. Akad. Nauk SSSR 4 (1935), 235-238. (Russian)

5. Y. Sagher, Hypersingular integrals with complex homogeneity, Ph.D. Thesis, Univ. of Chicago, 1967.

6. M. Weiss, On symmetric derivatives in $L^{p}$, Studia Math. 24 (1964), 89-100.

7. M. Weiss and A. Zygmund, On multipliers preserving convergence of trigonometric series almost everywhere, Studia Math. 30 (1968), 111-120.

8. A. Zygmund, Uber einige Sätze aus der Theorie der divergenten Reihen, Bull. Int. Acad. Polon. Sci. Lett. (1927), 309-331.

9. — , Trigonometric series, Vols. I, II, 2nd ed., Cambridge Univ. Press, New York, 1959.

UNIVERSITY OF MINNESOTA, MinNeAPOLIS, MinNeSOTA

UNIVERSITY OF ILLINOIS,

Chicago, Illinots 